Texture of Crystalline Solids, 1979, Vol. 3, pp. 245-254

0309-7951/79/0304-0245\$04.50/0

(c) 1979 Gordon and Breach Science Publishers, Inc.

Printed in Scotland

ANALYSIS OF ORIENTATION DISTRIBUTION PLOTS OBTAINED WITH THE VECTOR METHOD FOR CUBIC POLYCRYSTALS

D. RUER, A. VADON and R. BARO

Laboratoire de Métallurgie Structurale, Faculté des Sciences

57000 Metz, France

Abstract: The choice of the particular angular parameters $(\psi, \omega, \zeta)$ used in the vector method of texture analysis is justified and the relstionships for expressing any ideal orientation $(\psi, \omega, \zeta)$ in terms of (hkl) [uvw] and vice versa are given. Examples of orientation distribution are presented with constant sections of each of the three angular parameters.

\title{
INTRODUCTION
}

Although the theoretical basis of the vector method of texture analysis have already been published, $1-4$ it seems necessary to justify the choice of the parameters $\psi, \omega$ and $\zeta$ which, for different reasons, have been preferred to the traditional Euler's angles.

It seems therefore convenient to make available the relationships between these parameters and Miller's indices in order to allow the interpretation of the representation of the orientation distribution.

\section{JUSTIFICATION OF THE PARAMETERS}

The choice of the parameters was firstly imposed by the aim that was pursued in developing a new method of texture analysis. This aim was to elaborate a method which gives the best possible result utilising one pole figure only. In the particular case where the texture can be expressed by only one preferred orientation, it is obvious that one pole figure only is needed to find it. But, this is possible only if this single orientation can be related to all the angular parameters by one, and only one, set of three angular parameters.

Figure 1 shows that this condition is not satisfied by the use of Euler's angles. There are in fact 24 mathematically different orientations $\left(\varphi_{1}, \phi, \varphi_{2}\right)$ corresponding to a single physical orientation. Only two mathematically different orientations have been represented here as well as the unique possible mathematical orientation $(\psi, \omega, \zeta)$ corresponding to 


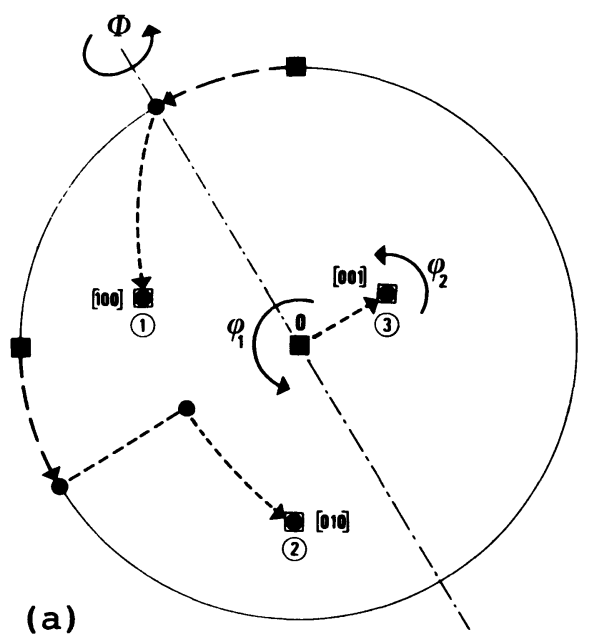

$\left(\varphi_{1}, \Phi, \varphi_{2}\right)=\left(30^{\circ}, 40^{\circ}, 50^{\circ}\right)$

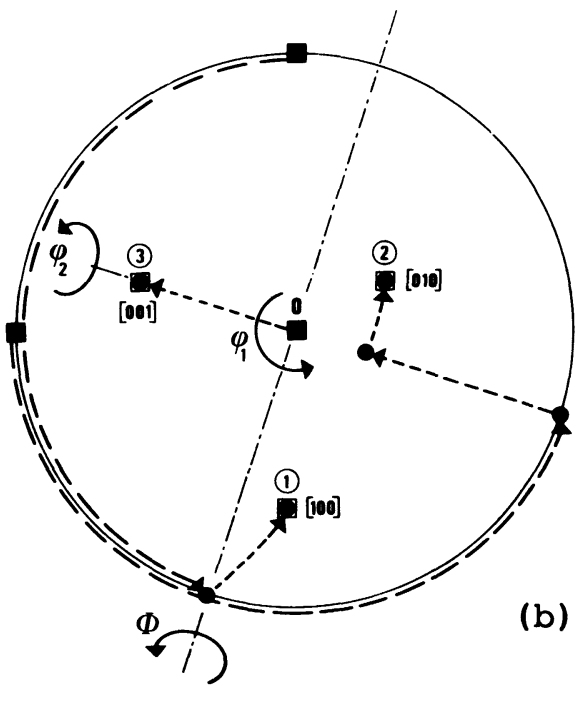

$\left(\varphi_{1}, \Phi, \varphi_{2}\right)=\left(162^{\circ}, 60^{\circ}, 28^{\circ}\right)$

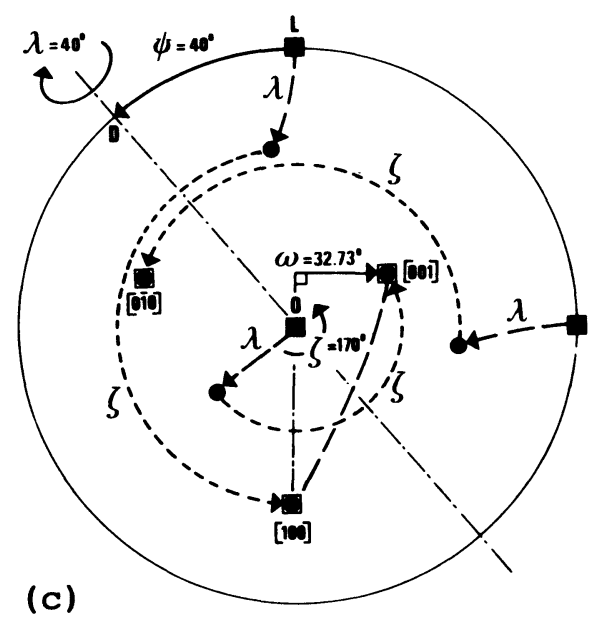

$$
(\psi, \lambda, \zeta)=\left(40^{\circ}, 40^{\circ}, 170^{\circ}\right)
$$

Figure 1. Representation of the same physical orientation: a) and b) with Euler's angles $\left(\varphi_{1}, \phi, \varphi_{2}\right)$; c) with the parameters $(\psi, \omega, \zeta)$.

the same physical orientation. Among these three parameters $\psi, \omega$ and $\zeta$, parameter $\omega$ plays a prominent part in permitting easily the restriction of the pole sphere to $1 / 24$ of it and thus giving the possibility to link together the 24 physically indistinguishable orientations with generally only one single set $(\psi, \omega, \zeta)$. 
REPRESENTATION OF THE ORIENTATION DISTRIBUTION

For reasons of readability it is usual to present the orientation distribution by lines of equal density plotted in sections corresponding to constant values of one of the three angular parameters. Although, in the vector method, it is theoretically indifferent to choose one constant parameter rather than another, the choice of the parameter $\zeta$ may seem preferable because, in this case, the sections are all equivalent to one of the two standard spherical triangles $T 1$ and T2 corresponding to the restriction of the pole sphere in the case of cubic crystal symmetry.

Figure 2 shows the orientation distribution of an aluminium sample rolled with 958 reduction rate. As this sample has no macroscopic symmetries it is here necessary to present the $\zeta$ sections for $\zeta$ varying from 0 to $\pm 2 \pi$. It must be kept in mind that parameters $\psi$ and $\zeta$ are both simultaneously negative or positive. This distribution was calculated from only one incomplete pole figure which is represented in Figure 3 with the corresponding recalculated one.

The points of each square correspond to the location of the normal of the sample inside the crystal reference system as shown in Figure 4 . If $\zeta$ has negative values, Miller's index $h$ must be replaced by $-h$. If the sample had presented orthorhombic symmetry, it would have been sufficient to take $\zeta$ varying between 0 and $\pi$. Indeed in this case, the orientations can be regrouped in the following manner: $(\psi, \omega, \zeta)$

equivalent to $(\psi, \omega, \zeta+\pi),(-\psi, \omega,-\zeta)$ and $(-\psi, \omega,-\zeta-\pi)$ as resulting from the macroscopic orthorhombic symmetry.

CORRESPONDENCE BETWEEN $(\psi, \omega, \zeta)$ and $(\mathrm{hkl})$ [uvw]

The main indices (hkl) of the crystal axis parallel to the sample normal can be directly read in the square $\zeta$ sections as shown in Figure 4. But to find the crystal axis parallel to the rolling direction, the following mathematical relationships are necessary:

$$
\begin{aligned}
\mathrm{h} & =\sin \psi \operatorname{tg} \omega\left(\cos ^{2} \psi+\operatorname{tg}^{2} \omega\right)^{-\frac{1}{2}} \\
-\mathrm{k} & =\cos \psi \operatorname{tg} \omega\left(\cos ^{2} \psi+\operatorname{tg}^{2} \omega\right)^{-\frac{1}{2}} \\
\mathrm{l} & =\cos \psi\left(\cos ^{2} \psi+\operatorname{tg}^{2} \omega\right)^{-\frac{1}{2}} \\
\mathrm{u} & =\left[\left(1+1-\mathrm{h}^{2}\right) \cos \zeta+\mathrm{hk} \sin \zeta\right](1+1)^{-1} \\
\mathrm{v} & =-\left[\left(1+1-\mathrm{k}^{2}\right) \sin \zeta+\mathrm{hk} \cos \zeta\right](1+1)^{-1} \\
\mathrm{w} & =-\mathrm{h} \cos \zeta+\mathrm{k} \sin \zeta
\end{aligned}
$$

In these relationships the following conditions have to be satisfied:

$$
\begin{aligned}
& \mathrm{h}^{2}+\mathrm{k}^{2}+\mathrm{1}^{2}=\mathrm{u}^{2}+\mathrm{v}^{2}+\mathrm{w}^{2}=1 \\
& |\psi| \in[0, \pi / 4], \omega \in[0, \pi / 4],|\zeta| \in[0,2 \pi] \text { and } \psi \cdot \zeta \geq 0
\end{aligned}
$$



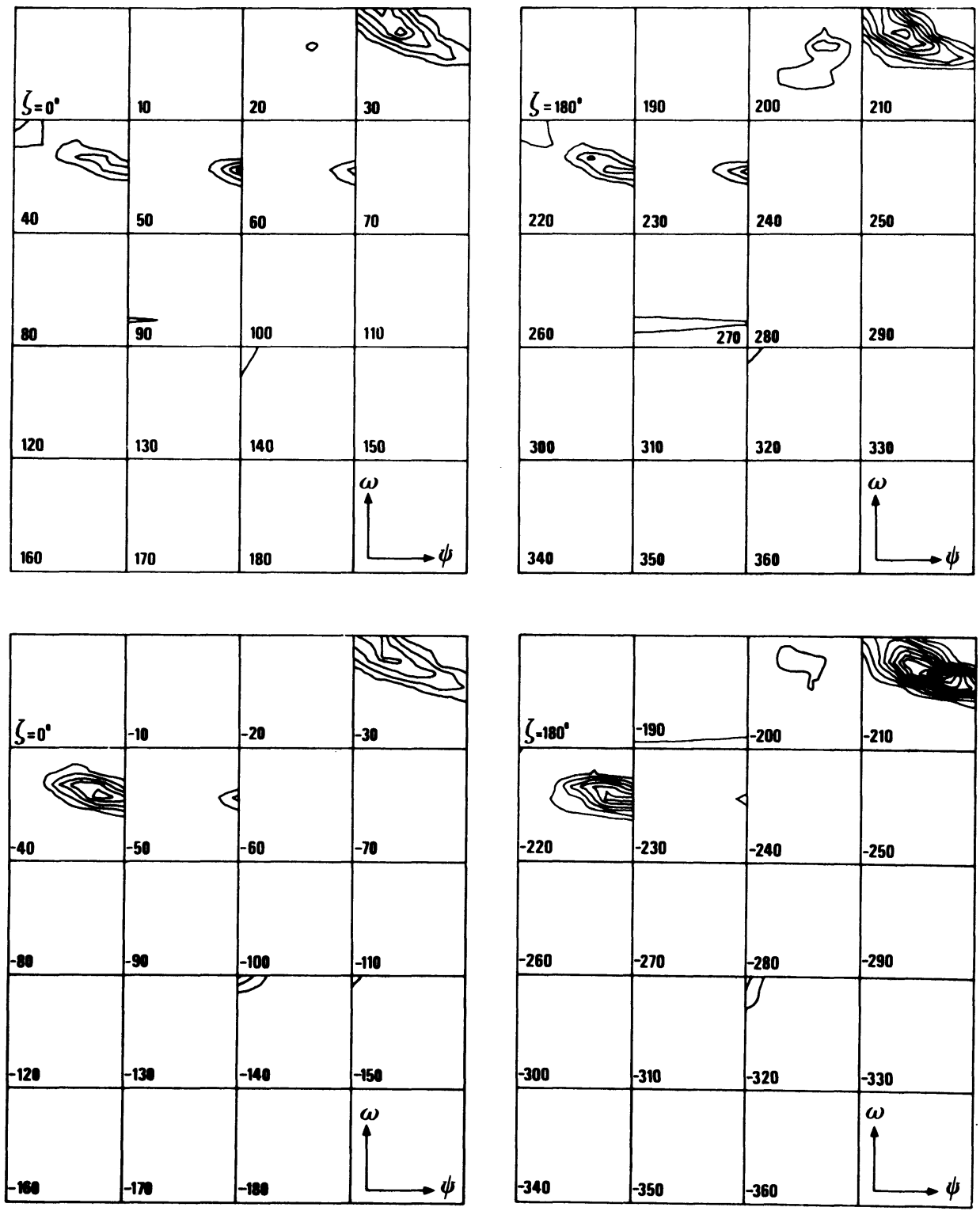

Figure 2. Orientation distribution of a rolled aluminium sheet having no macroscopic symmetry presented in constant $\zeta$ sections for $\zeta$ varying between 0 and $\pm 2 \pi$.

When one has to solve the inverse problem which consists in finding $\psi, \omega, \zeta$ knowing (hkl)[uvw], the following relationships give the solution.

$$
\begin{aligned}
& \sin \psi=h\left(h^{2}+k^{2}\right)^{-\frac{1}{2}} \text { or } \operatorname{tg} \psi=-h / k \\
& \sin \omega=-k\left(k^{2}+1^{2}\right)^{-\frac{1}{2}} \text { or } \operatorname{tg} \omega=-k / 1 \\
& \sin \zeta=-v+k w(1+1)^{-1} \\
& \cos \zeta=u-h w(1+1)^{-1}
\end{aligned}
$$




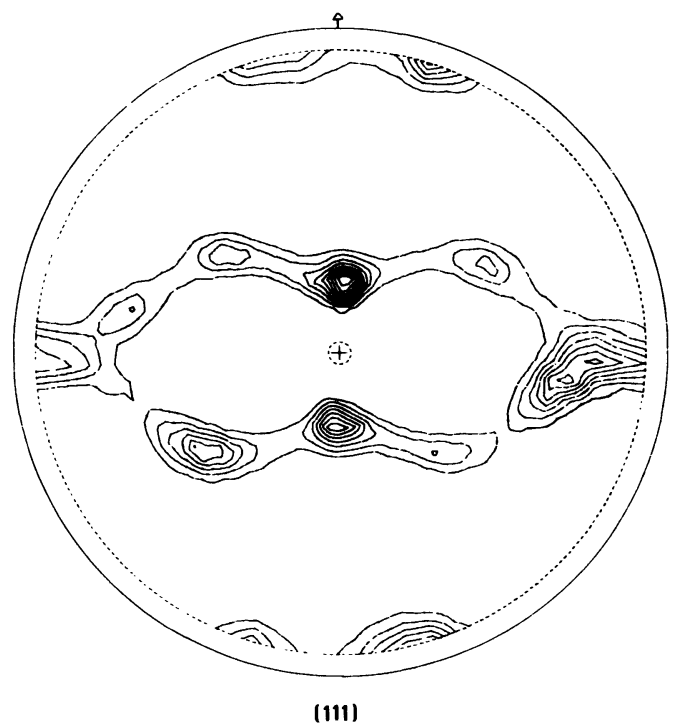

(a)

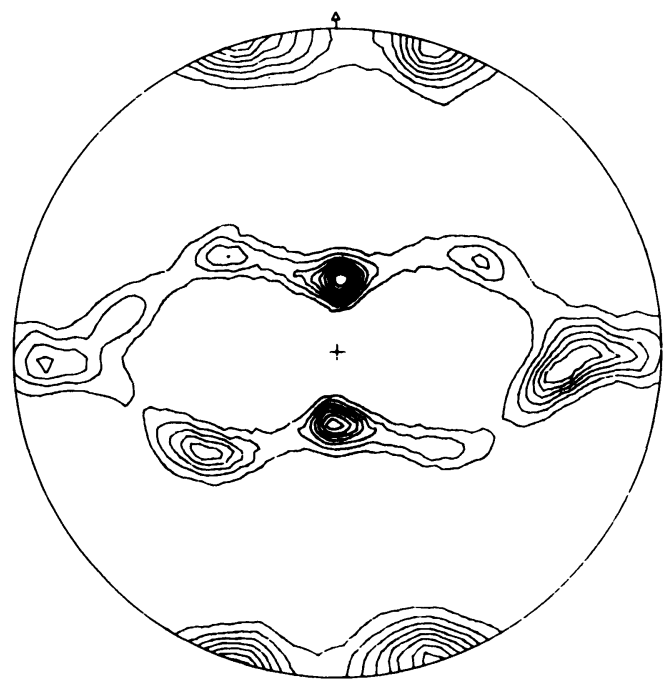

(b)

(111)

Figure 3. a) The single incomplete measured pole figure from which the distribution of Figure 2 was calculated. b) The corresponding recalculated complete pole figure. with the conditions

$$
\begin{aligned}
& \mathrm{h}^{2}+\mathrm{k}^{2}+\mathrm{l}^{2}=\mathrm{u}^{2}+\mathrm{v}^{2}+\mathrm{w}^{2}=1 \\
& \mathrm{k} \leq 0,1>0 \text { and }|\mathrm{h}|<|\mathrm{k}| \leq 1
\end{aligned}
$$

It must be pointed out that because $\zeta$ is included in the interval $[-2 \pi,+2 \pi]$ it is absolutely necessary to calculate sin $\zeta$ and cos $\zeta$. Furthermore it should not be forgotten that $\psi$ and $\zeta$ must always have the same sign. 


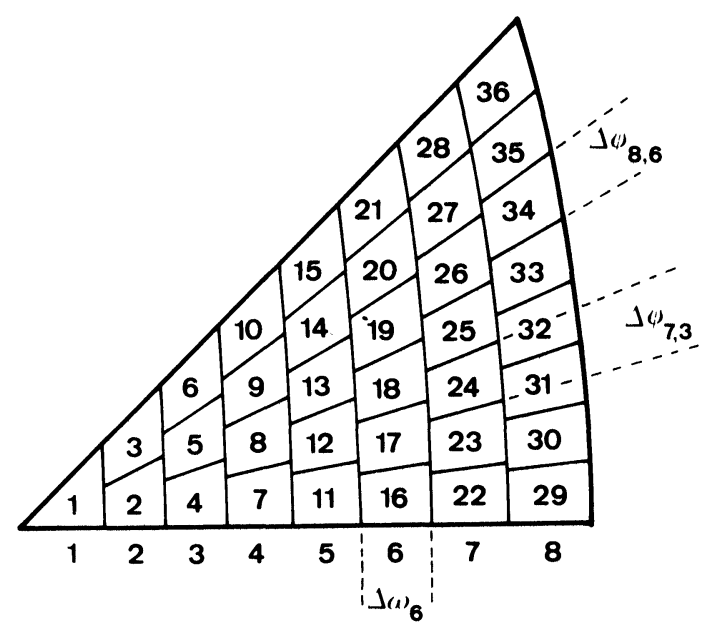

(a)

Triangle $T_{1}$

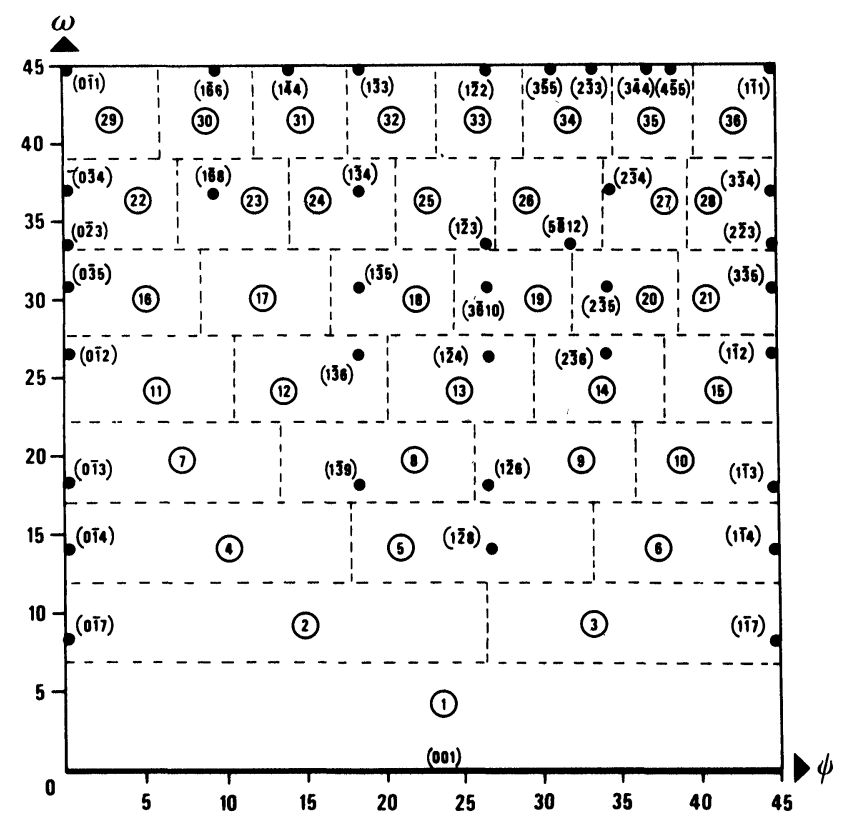

(b)

Figure 4. a) Triangle $T_{1}$ divided in 36 parts of equal spherical areas. b) Deformation of the triangle $T_{1}$ in a square constant $\zeta$ section (the point corresponding to (001) is replaced by a straight line segment).

In Table I the usual main orientations and the corresponding angular parameter values are tabulated.

We observe that for $(h k l)=(001)$ the parameter $\psi$ is arbitrary. 
TABLE I

Usual Ideal Orientations

\begin{tabular}{|c|c|c|c|c|}
\hline (hkl) & [ uvw] & $\psi$ & $\omega$ & $\zeta$ \\
\hline$(001)$ & 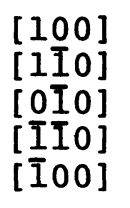 & & 0 & $\begin{array}{r}0 \\
45 \\
90 \\
135 \\
180\end{array}$ \\
\hline$(0 \bar{I} 1)$ & 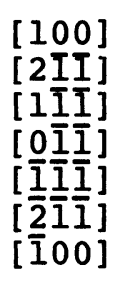 & 0 & 45 & $\begin{array}{l}0 \\
35.26 \\
54.74 \\
90 . \\
125.26 \\
144.74 \\
180 .\end{array}$ \\
\hline$(1 \overline{1} 1)$ & 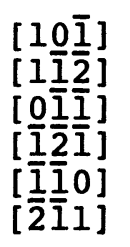 & 45 & 45 & $\begin{array}{l}15 . \\
45 \\
75 \\
105 \\
135 \\
165\end{array}$ \\
\hline$(1 \bar{I} 2)$ & $\begin{array}{l}{[3 \overline{1} \overline{2}]} \\
{[1 \overline{1} \overline{1}]} \\
{[\overline{1} \overline{3} \overline{2}]} \\
{[\overline{1} \overline{1} 0]} \\
{[\overline{3} \overline{1} 1]}\end{array}$ & 45 & 26.57 & $\begin{array}{l}22.79 \\
45 \\
67.21 \\
135 \\
166.48\end{array}$ \\
\hline
\end{tabular}

\section{DESCRIPTION IN PRESENCE OF ORTHORHOMBIC SYMMETRY}

In a sample having orthorhombic symmetry the orientations can be regrouped four by four. Each group of four symmetrically equivalent orientations corresponds to the parameter values $( \pm \psi, \omega, \pm \zeta \pm \pi)$ as already mentioned. But it is obvious that each group needs to be represented only once. It is then sufficient to limit parameter $\psi$ between 0 and $\pi / 4$ and parameter $\zeta$ between 0 and $\pi$. Hence each group of four symmetrically equivalent orientations will appear in the description generally only once.

Figure 5 shows the orientation distribution of a rolled mild steel sample having orthorhombic symmetry. This distribution is successively presented in constant $\zeta, \psi$ and $\omega$ sections. It can be seen that the use of constant $\psi$ or $\omega$ sections gives a better view upon the whole distribution, as shown in Figure 6 .

Furthermore, in this example, the restriction of parameters $\psi$ and $\omega$ in the interval $[\pi / 6, \pi / 4]$ would suppress only a 


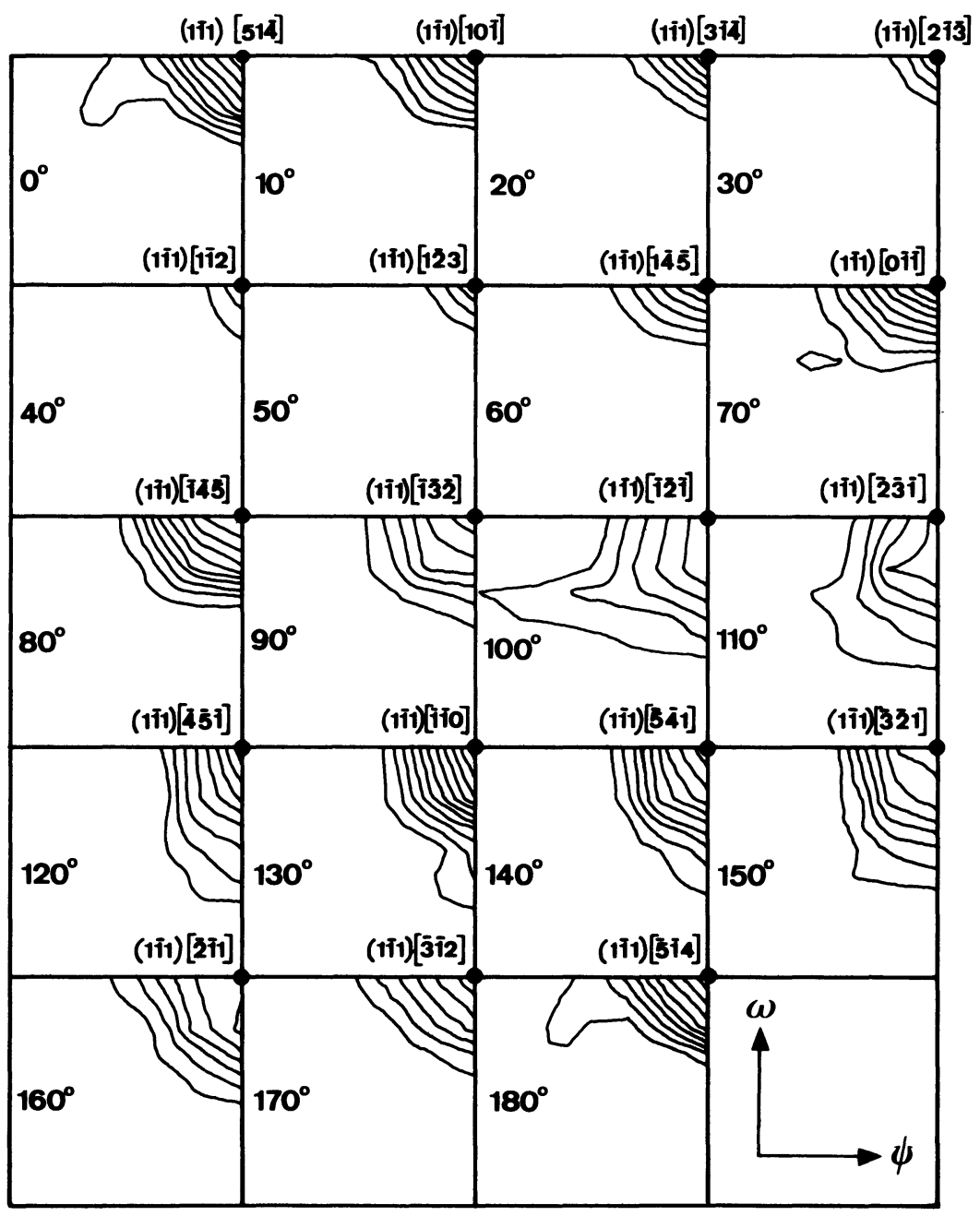

Figure 5. Orientation distribution of a rolled mild steel sheet having orthorhombic macroscopic symmetry presented in constant $\zeta$ sections.

negligible part of the distribution. Whatever parameter is used, it appears clearly that the texture of this mild steel sheet is a (111) fiber texture with maxima corresponding to $\{111\}<110>$.

\section{CONCLUSION}

In this paper we give the mathematical expressions that are needed for interpreting the orientation distribution presented in constant parameter sections in the vector method.

Parameter $\omega$ used in the vector method allows the restriction of the pole sphere to the minimal part needed that is to say, to two spherical standard triangles only.

The consequence is an adequate limitation of the parameter values that minimizes the redundancies of physical 


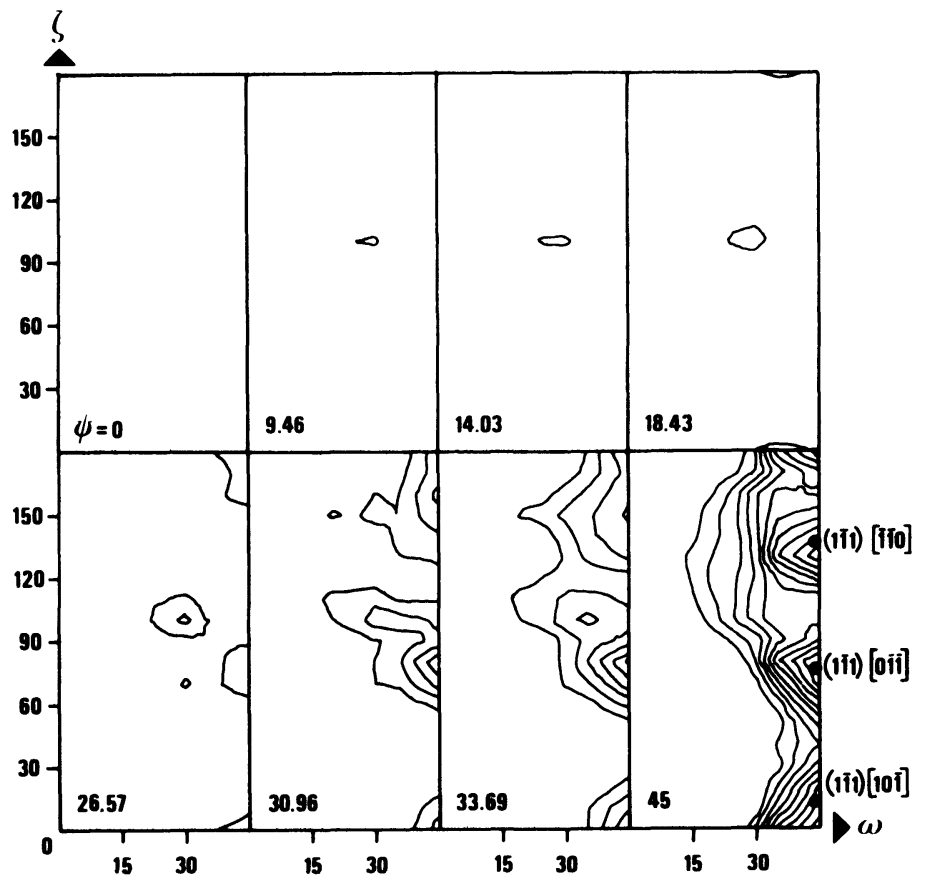

(a)

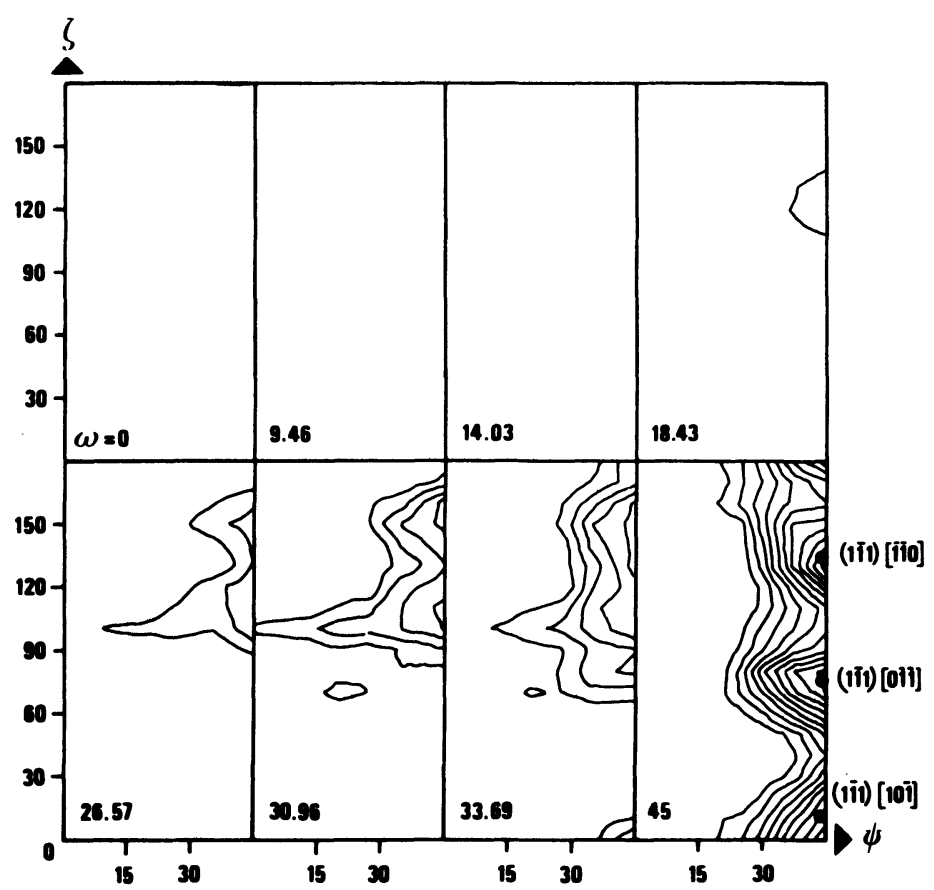

(b)

Figure 6. The same distribution as in Figure 5 but presented a) in constant $\psi$ sections; b) in constant $\omega$ sections. 
equivalent orientations in the plotting of an orientation distribution.

\section{ACKNOWLEDGMENT}

We express our thanks to Dr. Lücke and Dr. Virnich of the University of Aachen for the communication of the experimental data represented in Figure $3 a$.

\section{REFERENCES}

1. D. Ruer, Thèse d'Etat (1976).

2. D. Ruer and R. Baro, Advances in X-Ray Analysis, 20, 187 (1977).

3. D. Ruer and R. Baro, J. Appl. Cryst., 10, 458 (1977).

4. D. Ruer and R. Baro, Mém. Sc. Rev. Métall. (to be published). 